$4 \quad$ ABSTRACT

\title{
Interpretation of Cone Penetration Test Data in Layered Soils using Cavity Expansion Analysis
}

\author{
Pin-Qiang Mo ${ }^{1}$, Alec M. Marshall ${ }^{2}$, and Hai-Sui $\mathrm{Yu}^{3}$
}

Cavity expansion theory plays an important role in many geotechnical engineering problems, including the cone penetration test (CPT). One of the challenges of interpreting CPT data is the delineation of interfaces between soil layers and the identification of distinct thin layers, a process which relies on an in-depth understanding of the relationship between penetrometer readings and soil properties. In this paper, analytical cavity expansion solutions in two concentric regions of soil are applied to the interpretation of CPT data, with specific focus on the layered effects during penetration. The solutions provide a large-strain analysis of cavity expansion in two-concentric regions for dilatant elastic-perfectly plastic material. The analysis of CPT data in two-layered soils highlights the effect of respective soil properties (strength, stiffness) on CPT measurements within the influence zones around the two-soil interface. Results show good comparisons with numerical results and elastic solutions. A simple superposition method of the two-layered analytical approach is applied to the analysis of penetration in multi-layered soils. A good comparison with field data and numerical results is obtained. It is illustrated that the proposed parameters effectively capture the influence of respective soil properties in the thin-layer analysis. It is also shown that results based on this analysis have better agreement with numerical results compared with elastic solutions.

Keywords: cone penetration test, layered soils, cavity expansion analysis.

\footnotetext{
${ }^{1}$ Assistant Research Scientist, State Key Laboratory for GeoMechanics and Deep Underground Engineering, China University of Mining \& Technology. Former Research Fellow at University of Nottingham. E-mail: mopinqiang@gmail.com; pinqiang.mo@cumt.edu.cn

${ }^{2}$ Assoc. Prof., Faculty of Engineering, University of Nottingham, UK. E-mail: alec.marshall@nottingham.ac.uk

${ }^{3}$ Prof., Faculty of Engineering, University of Nottingham, UK. E-mail: hai-sui.yu@nottingham.ac.uk
} 


\section{INTRODUCTION}

The cone penetration test $(\mathrm{CPT})$ is a proven tool for in situ soil testing. The test method can provide data for evaluation of important geotechnical design parameters, delineation of different soil profiles within the ground, calculation of end-bearing capacity of piles, and assessment of liquefaction potential. There are a number of methods available for the analysis and interpretation of CPT data, as discussed in Yu and Mitchell (1998), which include bearing capacity theory, steady state approaches, empirical relationships based on experimental tests, numerical analysis, and cavity expansion theory. The focus of this paper is on the use of cavity expansion theory for interpretation of CPT data in layered soils.

Cavity expansion theory has been applied to the analysis of many engineering problems. One of its first applications was for the analysis of the indentation of ductile materials (Bishop et al. 1945). For geotechnical application, Gibson and Anderson (1961) adopted the theory of cylindrical cavity expansion for the estimation of soil properties from pressuremeter test data. Thereafter, numerous analytical and numerical solutions have been proposed using increasingly sophisticated constitutive soil models. The development of the theory and its application to geomechanics was described in detail in $\mathrm{Yu}$ (2000). The application of cavity expansion analyses to penetration problems was first reported by Bishop et al. (1945) who noted that the penetrating force is proportional to cavity expansion pressure. Since that time, a considerable amount of research has been carried out to improve the theoretical solutions relating to cavity pressure (particularly the limit pressure) and to investigate the correlation between the cavity pressure and penetrometer resistance. Cone penetration certainly involves more than a single mechanism, such as either cylindrical or spherical cavity expansion. As pointed out by $\mathrm{Yu}$ (2006) in his Mitchell Lecture, cone penetration can be modelled by three different ways using cavity expansion theory. They include a spherical cavity expansion approach (e.g. Vesic 1977), a cylindrical cavity approach (e.g. Salgado et al. 1997), and a combined cylindrical-spherical cavity expansion approach (Yu 2006). For each approach, a different correlation would need to be used to approximate cone penetration using cavity 
expansion solutions. Based on precedence of other researchers, the spherical cavity expansion analysis was considered to be more appropriate for this study due to its reasonable replication of the displacement patterns near the penetrometer tip and the available correlations between spherical cavity expansion pressure and penetration resistance, which this paper relied on.

Despite the wide application of the theory to geotechnical problems, very little work has been done to consider the effect of distinct soil layers within the framework of cavity expansion analyses. Sayed and Hamed (1987) were the first to apply analytical cavity expansion analyses of concentrically layered media to the field of geomechanics. They applied an elastic solution for spherical expansion to evaluate pile settlement in soil layers, and a cylindrical analysis was used to investigate the effect of a remoulded annulus on the stress-strain behavior and deformation response of the intact soil. Xu and Lehane (2008) used a numerical analysis of spherical cavity expansion to investigate pile or probe resistance in two-layered soil profiles using a nonlinear elastic hardening soil model. Mo et al. (2014) provided the first analytical solutions of cavity expansion in two concentric regions for dilatant elasticperfectly plastic material, using a Mohr-Coulomb yield criterion, a non-associated flow rule, and a large-strain analysis.

The results presented by Mo et al. (2014) illustrated that the cavity expansion method can be used to study problems involving two concentric regions of soil. The purpose of this paper is to illustrate that the analytical solutions of Mo et al. (2014) can be effectively applied to the interpretation of CPT data in two-layered as well as multi-layered soils. The advantage of the analytical method over numerical and experimental methods is that it provides a more efficient tool for studying the problem. There are numerous examples of numerical and experimental analyses of CPT tip resistance or pile end bearing capacity in layered soils (Xu and Lehane 2008, Ahmadi and Robertson 2005, Mo et al. 2013, 2015) and multi-layered soils (Hird et al. 2003, Ahmadi and Robertson 2005, Walker and Yu 2010), from which some useful data are used in this paper for validation of the proposed analytical method. 
The paper is organized into four main sections. The correlation between concentric and horizontal layering is provided first, aiming to reveal the analogue between cavity expansion in concentric soils and cone penetration in horizontally layered soils. After illustrating the combination method to relate the theoretical model to the penetration problem, cone tip resistance during penetration in layered soils is investigated using the analytical solutions. The layered and thin-layer effects on penetration resistance are then studied using the analytical solutions, with some parametric studies also provided. Results of interpretation of CPT measurements are then compared with numerical results from the literature.

\section{CONCENTRIC AND HORIZONTAL LAYERING}

The use of cavity expansion in concentric media as an analogue to cone penetration in horizontal soil layers is discussed in this section. For theoretical solutions, an infinite medium or circular/spherical boundary is generally preferred since the symmetric boundary conditions simplify the solutions significantly. Equivalently, most cavity expansion methods employ similar boundary assumptions. A direct application of a concentrically layered model of cavity expansion to pile foundations was proposed by Sayed and Hamed (1987) using elastic analyses. The comparison of cavity expansion in concentric regions and cone penetration in horizontal layers is shown in Fig. 1.

In order to study the differences between cavity expansion in concentrically and horizontally layered models, numerical simulations using Abaqus/Standard were conducted. A schematic of the two models is shown in Fig. 2, where an axisymmetric model was used to provide the spherical cavity expansion analysis. The cavities were expanded from an initial radius of $a_{0}=6 \mathrm{~mm}$ under an initial isotropic pressure of $P_{0}=1 \mathrm{kPa}$ by increasing the cavity pressure, $P_{a}$. The size of the two-soil interface $b_{0}$ varied from $a_{0}$ to infinity. The analogy presented in Fig. 2b considers penetration from Soil 1 (weaker soil) into Soil 2 (stronger soil). Note that the terms weak and strong are used throughout the paper to indicate not only relative strength of materials but also stiffness. A non-associated Mohr-Coulomb soil model was used for the analytical solutions, as described in Mo et al. (2014), where the plastic flow 
rule assumes the soil dilates plastically at a constant rate. In general, drained behaviour of sand could be accurately modelled by the non-associated Mohr-Coulomb model, while the perfect plasticity indicates the strength of material remains constant during loading and unloading. Five parameters are required to represent the soil stress-strain relationship: Young's modulus $(E)$, Poisson's ratio $(\nu)$, friction angle $(\phi)$, cohesion $(C)$ and dilation angle $(\psi)$. The soil parameters were set as follows: $\nu=0.2, \phi=10^{\circ}, \psi=10^{\circ}, C=10 \mathrm{kPa}$; $E_{\text {Soil } 1}=1 M P a$ and $E_{\text {Soil } 2}=10 M P a$.

The penetration process in the concentric model was simulated in two stages corresponding to the states when the cone tip was located within the two different soils. The cone tip starts within the weaker Soil 1 and approaches the stronger Soil 2. The approach of the cone tip towards the soil interface is simulated by decreasing $b_{0}$ from $\infty$ to $a_{0}$ with Soil $\mathrm{A}=$ Soil 1 and Soil B = Soil 2 (Fig. 2a). The cone tip then enters the stronger soil, and the reversal of Soil A and Soil B is required for the concentric model, hence Soil A = Soil 2 and Soil B = Soil 1. Movement of the cone tip away from the interface is simulated by increasing $b_{0}$ from $a_{0}$ to $\infty$. The cavity expansion in the horizontal model (Fig. 2b) is simulated correspondingly by moving the position of the soil interface, given by $b_{0}$.

A comparison between the two model results of cavity pressure with variation of the soil interface $\left(b_{0} / a_{0}\right)$ at an expansion stage of $a / a_{0}=1.2$ is illustrated in Fig. 3 (' $a$ ' refers to the radius of cavity after expansion). The two horizontal reference lines are the cavity pressures required to achieve this expansion stage in uniform weak and strong soils. The horizontally layered soil model provides a smooth transition of cavity pressure (and implied penetration resistance) from one layer to the next. The cavity pressures from the concentrically layered numerical model don't show a smooth transition across the interface but instead range from the uniform soil extremes on each side of the interface. The size of the influence zones around the interface is related to the stiffness and strength of the respective soil layers, as demonstrated by the results from both the concentrically and horizontally layered models. Included in Fig. 3 is a transition line based on a proposed combination method in which 
the concentrically layered results are used to provide a transition curve which is comparable to that obtained from the horizontally layered model. The combination method will be explained later in the following section.

It should be noted that the Mo et al. (2014) analytical solution gives exactly the same results as the concentric numerical model for the same model conditions. A limitation of the numerical simulation of this problem is that the degree of cavity expansion is limited by the allowable level of distortion of the numerical soil elements. The results presented in Fig. 3 are therefore based on the simulation of a relatively small expansion ratio of $a / a_{0}=1.2$. The analytical method, on the other hand, can provide precise solutions for expansion to an arbitrary size (Mo et al. 2014), thereby improving the applicability of the method.

\section{PENETRATION IN TWO-LAYERED SOILS}

\section{Combination Method}

The limit pressure is often applied to predict pile capacity or probe resistance in conventional cavity expansion solutions (e.g. Randolph et al. 1994). This approach is appropriate for uniform soils since the limiting pressure is only affected by the parameters of a single soil layer. In layered soils, the results in Mo et al. (2014) showed that the limiting pressure depends only on the properties of Soil B (the outer layer or the lower layer). For penetration problems such as CPT or pile capacity analysis, the resistance of a probe located in Soil A depends in part on the properties of Soil A (refer to Fig. 4), so the limit pressure approach is not adequate for layered soils. A more suitable approach for layered soils, as suggested by $\mathrm{Xu}$ and Lehane (2008), is to consider a realistic increase in cavity size (given by $a / a_{0}$ ) and evaluate the cavity pressure required to achieve this expansion. Therefore, to investigate cone tip resistance $\left(q_{c}\right)$ in layered soils, the cone penetration process at a given depth is modeled as a spherical cavity expanded slowly from an initial diameter close in size to the average grain size of the soil to a final size corresponding to the diameter of the penetrometer (i.e. $a=B / 2$ ). The cone tip resistance is then related to the corresponding cavity pressure that is calculated, as depicted in Fig. 4. The penetration process is simulated by first considering an analysis 
point in Soil A (a weaker soil) sufficiently far away from the Soil A/B interface such that Soil B has no effect, then considering points increasingly close to the interface, and finally moving into Soil B (a stronger soil). The distance to the soil interface is defined as $H$, which is equivalent to $b_{0}$ in the cavity expansion analysis.

As $b_{0}$ decreases from infinity to $a_{0}$ (i.e. the cone tip approaches the interface between Soil A and Soil B), cavity pressure $\left(P_{a}\right)$ transforms from $P_{a, A}$ to $P_{a, B}$ (see to Fig. 5 where the subscripts $w$ and $s$ refer to the layers of weaker and stronger soil). The cavity pressure variation during this process provides the transition from Soil A to Soil B (dashed lines in Fig. 5). However, these two lines do not give an adequate description of the transition of cavity pressure $P_{a}$ between the soil layers, owing to the two extremes at the soil interface (as discussed earlier for the data in Fig. 3). To overcome this deficiency, the lines need to be combined to provide an interpolated transition of cavity pressure, $P_{a, t r}$. A simple combination approach for the scenario of a weaker soil overlying a stronger soil is provided in Fig. 5, which is based on the secant angles $\left(\theta_{1}\right.$ and $\left.\theta_{2}\right)$ at points located $1 B$ from the interface (i.e. a straight line on each side of the interface formed by two points at $|H|=0$ and $|H|=B)$. The modified cavity pressure at the interface $\left(P_{a, i n t}\right)$ is calculated by:

$$
\frac{P_{a, i n t}-P_{a, w}}{P_{a, s}-P_{a, i n t}}=\frac{\tan \theta_{1}}{\tan \theta_{2}}
$$

and the transitionary cavity pressure curve $\left(P_{a, t r}\right)$ is obtained by:

$$
P_{a, t r}= \begin{cases}P_{a, w}+\left(P_{a}-P_{a, w}\right) \times \frac{P_{a, i n t}-P_{a, w}}{P_{a, s}-P_{a, w}} & \text { (cavity in weak soil) } \\ P_{a, s}-\left(P_{a, s}-P_{a}\right) \times \frac{P_{a, s}-P_{a, i n t}}{P_{a, s}-P_{a, w}} & \text { (cavity in strong soil) }\end{cases}
$$

A cavity pressure ratio is defined as $\eta_{P_{a}}^{\prime}=\left(P_{a, t r}-P_{a, w}\right) /\left(P_{a, s}-P_{a, w}\right)$ to represent the transition from weak soil $\left(\eta_{P_{a}}^{\prime}=0\right)$ to strong soil $\left(\eta_{P_{a}}^{\prime}=1\right)$. Ultimately, the prediction of cone tip resistance is needed. The correlations for calculating cone tip resistance from spherical cavity pressure in cohesionless and cohesive soils proposed by Yasufuku and Hyde 
(1995) and Ladanyi and Johnston (1974), respectively, were used to estimate $q_{c}$ in this analysis:

$$
q_{c}= \begin{cases}P_{a, t r} /\left(1-\sin \phi_{\text {smooth }}\right) & (\text { cohesionless soils }) \\ P_{a, t r}+\sqrt{3} s_{u, \text { smooth }} & \text { (cohesive soils) }\end{cases}
$$

where $\phi_{\text {smooth }}$ and $s_{u, \text { smooth }}$ are 'smoothed' friction angle and undrained shear strength, respectively. Mathematically, the correlation between cavity pressure and penetration resistance is a function of soil properties, therefore a smooth transition of soil properties is required to obtain a smooth curve of penetration resistance. The ratio $\eta_{P_{a}}^{\prime}$ is used to smooth the transition of soil properties within the analysis (e.g. $\left.\phi_{s m o o t h}=\phi_{w}+\eta_{P_{a}}^{\prime} \times\left(\phi_{s}-\phi_{w}\right)\right)$. The transition of cone tip resistance, $q_{c}$, from the weaker to the stronger soil can then be obtained by combining the data from Eq. 1 and 2 into Eq. 3.

To evaluate layered effects on the resistance of penetrometers, $\mathrm{Xu}$ and Lehane (2008) performed a series of numerical analyses of spherical cavity expansion and proposed a resistance ratio, defined as $\eta=q_{c} / q_{c, s}$, based on a parametric study which was also validated against centrifuge tests. The influence zone in the stronger layer was defined as the location where $\eta=0.95$, whereas in the weaker layer it was defined as the location where $\eta=0.05+0.95 \eta_{\min }$.

A modified cone tip resistance ratio, $\eta^{\prime}$, is proposed here as:

$$
\eta^{\prime}=\frac{q_{c}-q_{c, w}}{q_{c, s}-q_{c, w}}
$$

which like $\eta_{P_{a}}^{\prime}$ also varies from 0 to 1 corresponding to the transition from a relatively weak to strong soil. The influence zones within the weaker and stronger soil layers, referred to as $Z_{w}$ and $Z_{s}$, respectively, are defined as areas where $0.05<\eta^{\prime}<0.95$.

The newly defined resistance ratio (Eq. 4) can also be related to the resistance ratio proposed by $\mathrm{Xu}$ and Lehane $(2008)$ as $\eta^{\prime}=\left(\eta-\eta_{\min }\right) /\left(1-\eta_{\min }\right)$. This allows a direct comparison of the expression obtained in $\mathrm{Xu}$ and Lehane (2008), based on their regression 
analysis of the numerical model results, to the new resistance ratio $\eta^{\prime}$, using the following expression:

$$
\eta^{\prime}=\exp \left[-\exp \left(B_{1}+B_{2} \times H / B\right)\right]
$$

where $B_{1}=-0.22 \ln \left(q_{c, w} / q_{c, s}\right)+0.11 \leq 1.5$ and $B_{2}=-0.11 \ln \left(q_{c, w} / q_{c, s}\right)-0.79 \leq-0.2$.

\section{Interpretation of results}

A series of cavity expansion simulations in two-layered soils was carried out to explore the effect of changing soil relative density $\left(D_{R}\right)$ across an interface. The simulations considered an initial condition of constant confining stress in order to replicate the environment in a calibration chamber test with no boundary effects. A value of $P_{0}=1 k P a$ was used in the simulations. The soil model parameters for different values of $D_{R}$ are provided in Table 1 using the approach presented in Appendix 1, with estimated values of cone resistance in a uniform soil layer based on a penetrometer with a diameter of $12 \mathrm{~mm}$ using Eq. 3 .

By varying the relative density of each soil layer, the cone tip resistance and resistance ratio curves shown in Fig. 6 and 7, respectively, were obtained (using the relationship from Yasufuku and Hyde 1995). The transformation curves are plotted against the normalised distance to the interface $(H / B)$ and show that the influence zone in the stronger layer is larger than in the weaker soil, which agrees with experimental observations (Xu and Lehane 2008, Mo et al. 2015) and field tests (Meyerhof and Sastry 1978a, 1978b, Meyerhof 1983).

The studies of Meyerhof $(1976,1977)$ suggested a constant size influence zone around the soil interface: $10 B$ in dense sand, and $2 B$ in loose sand. A linear transition is generally used for pile design. However, from the resistance ratio curves presented, the transition zones on both sides of the soil interface are shown to be non-linearly dependent on the properties of both soil layers. The size of the influence zones varies with the relative density of the soil layer; it can be seen that $Z_{w}$ increases with an increase of relative density of the weaker soil and that $Z_{s}$ increases with an increase of relative density of the stronger soil. The size of the influence zone in a soil layer is also affected by the relative density of the adjacent soil; the 
size of $Z_{w}$ is shown to decrease with an increase of relative density of the stronger soil and the size of $Z_{s}$ is shown to decrease with an increase of relative density of the weaker soil.

It was found that the size of the influence zones in the stronger and weaker zones could be effectively related to the relative densities of the two soils: $D_{R, w}$ and $D_{R, s}$. The data of $Z_{s}$ and $Z_{w}$, normalized by $B$, are plotted against $D_{R, w}$ and $D_{R, s}$ in Fig. 8. A surface was fitted to the data to provide expressions of normalized influence zones, as illustrated in Fig. 8 and given by:

$$
\begin{aligned}
& Z_{w} / B=-0.0871 \times D_{R, w}+0.0708 \times D_{R, s}-5.8257 \\
& Z_{s} / B=-0.1083 \times D_{R, w}+0.1607 \times D_{R, s}+5.1096
\end{aligned}
$$

where $D_{R}$ is in '\%'. The correlation coefficients $R^{2}$ are 0.9639 and 0.9955 , respectively. The expressions are only valid for this particular soil in a certain stress condition, however they imply a linear relationship between influence zone size and relative density.

\section{Comparison with numerical results and elastic solutions}

This section compares results obtained using the cavity expansion analysis proposed in this paper against other data available for penetration resistance in layered soils. Fig. 9 compares $\eta^{\prime}$ values obtained from the various sources (discussed below) for equivalent soil properties and stress conditions. The data illustrate that the results from this study compare very well with other published methods.

The data from Ahmadi and Robertson (2005) is based on a numerical model of cone tip resistance in layered soils using a Mohr-Coulomb elastic-plastic material. The results of $\eta^{\prime}$ from two of their tests are plotted in Fig. 9: (a) loose sand $\left(D_{R}=30 \%\right)$ overlying dense sand $\left(D_{R}=90 \%\right)$; (b) soft clay $\left(s_{u}=20 \mathrm{kPa}\right)$ overlying dense sand $\left(D_{R}=90 \%\right)$. Note that the undrained behaviour of clay was modelled with the Mohr-Coulomb model by setting Poisson ratio close to 0.5 with a stress independent shear modulus of $6 \mathrm{MPa}$ and cohesion (undrained shear strength) of $20 \mathrm{kPa}$. Model parameters were consistent with those from van den Berg (1994), Ahmadi et al. (2005) and Ahmadi and Robertson (2005), who used them for numerical analysis using FLAC. 
The results of Xu and Lehane (2008) were determined using Eq. 5, which was proposed according to the numerical simulation of cavity expansion. The lines of Vreugdenhil et al. (1994) are based on an approximate elastic analysis for interpretation of cone penetration results in multi-layered soils where the CPT is represented by a circular uniform load. The resistance ratio curve is solely dependent on the stiffness ratio, and this ratio here is approximated as $q_{c, w} / q_{c, s}$ for comparison. Note that $q_{c}$ for clay was determined based on the relationship of Ladanyi and Johnston (1974), as presented in Eq. 3.

\section{PENETRATION IN MULTI-LAYERED SOILS}

The cone penetration resistance in multi-layered soils can be obtained by superposition of resistance ratios $\left(\eta^{\prime}\right)$ in two-layer systems. When a cone tip is in a thin 'sandwiched' soil layer, the cone tip resistance becomes affected by the subsequent soil layer before it reaches a steady-state resistance in the thin soil layer. Hence, interpretation of CPT data in thin layers may easily over- or under-predict soil properties. The effects of thin layer thickness and soil properties are investigated in this section.

\section{Analysis Methodology}

Figure 10 describes the cone penetration process in multi-layered soils where a strong soil is embedded within a weak soil (assuming the layers of weak soil have the same properties). A similar scenario of weak soil embedded in strong soil can also be considered. When the thickness of the strong soil $\left(H_{t}\right)$ is thin enough $\left(<2 Z_{s}\right)$, the maximum achieved cone tip resistance $\left(q_{c, \max }\right)$ is less than the resistance in the uniform strong soil $\left(q_{c, s}\right)$. The maximum resistance is affected by the influence zones $\left(Z_{w}\right.$ and $\left.Z_{s}\right)$ and the thickness of the strong soil $\left(H_{t}\right)$. A schematic profile of cone tip resistance ratio $\left(\eta^{\prime}\right)$ in the thin-layer of strong soil is shown in Fig. 11a, with definition of maximum resistance ratio $\left(\eta_{\max }^{\prime}\right)$. For the scenario of a thin-layer of weak soil in Fig. 11b, penetration resistance in the strong soil $\left(\eta^{\prime}=1\right)$ is influenced by the weak layer, and the thin-layer effect is evaluated by the minimum resistance ratio $\left(\eta_{\text {min }}^{\prime}\right)$. The difference between the peak resistance ratio and the uniform value provides 
a measure of the thin-layer effect (i.e. $1-\eta_{\max }^{\prime}$ for the thin strong layer and $\eta_{\min }^{\prime}-0$ for the thin weak layer).

From the analytical solution in two-layered soils presented in the previous section, the resistance ratio for multi-layered soils can be obtained by superposition of $\eta^{\prime}$ in multiple twolayered profiles. For example, when the strong soil is sandwiched by two layers of weak soil, the profile is a combination of 'weak-strong' (subscript ws) and 'strong-weak' (subscript sw), with resistance ratios of $\eta_{w s}^{\prime}=\eta^{\prime}(H)$ and $\eta_{s w}^{\prime}=\eta^{\prime}\left(H_{t}-H\right)$. This is based on a symmetric assumption, $\left.\eta_{w s}^{\prime}\right|_{H=0}=\left.\eta_{s w}^{\prime}\right|_{H=H_{t}}$ and $\left.\eta_{w s}^{\prime}\right|_{H=H_{t} / 2}=\left.\eta_{s w}^{\prime}\right|_{H=H_{t} / 2}$. When simply multiplying the resistance ratios, the maximum resistance ratio equals $\left(\left.\eta_{w s}^{\prime}\right|_{H=H_{t} / 2}\right)^{2}$, and varies from $\left(\left.\eta_{w s}^{\prime}\right|_{H=0}\right)^{2}$ to 1 when increasing the thickness of the sandwiched soil layer $\left(H_{t}\right)$ from 0 to infinity. In order to eliminate this inconsistency, a correction factor $\left(\chi_{w s w}\right)$ is integrated within the superposition of $\eta_{w s}^{\prime}$ and $\eta_{s w}^{\prime}$. The generated resistance ratio and the maximum resistance ratio in the three-layered system with a thin layer of strong soil are expressed as:

$$
\eta^{\prime}=\eta_{w s}^{\prime} \times \eta_{s w}^{\prime} \times \chi_{w s w}
$$

$$
\eta_{\text {max }}^{\prime}=\left(\left.\eta_{w s}^{\prime}\right|_{H=H_{t} / 2}\right)^{2} \times \chi_{w s w}
$$

where

$$
\chi_{w s w}=\frac{\left(\left.\eta_{w s}^{\prime}\right|_{H=H_{t} / 2}\right)^{2}-\left(\left.\eta_{w s}^{\prime}\right|_{H=0}\right)^{2}}{1-\left(\left.\eta_{w s}^{\prime}\right|_{H=0}\right)^{2}}
$$

Correspondingly, the system with a thin layer of weak soil can be produced in a similar process for the calculation of $\eta_{\text {min }}^{\prime}$ using:

$$
\eta^{\prime}=1-\left(1-\eta_{s w}^{\prime}\right) \times\left(1-\eta_{w s}^{\prime}\right) \times \chi_{s w s}
$$

$$
\eta_{\text {min }}^{\prime}=1-\left(1-\left.\eta_{s w}^{\prime}\right|_{H=H_{t} / 2}\right)^{2} \times \chi_{s w s}
$$


where

$$
\chi_{s w s}=\frac{\left(1-\left.\eta_{w s}^{\prime}\right|_{H=H_{t} / 2}\right)^{2}-\left(1-\left.\eta_{w s}^{\prime}\right|_{H=0}\right)^{2}}{1-\left(1-\left.\eta_{w s}^{\prime}\right|_{H=0}\right)^{2}}
$$

\section{Thin-layer analysis results}

Strong soil within weak soil

Fig. 12 shows the resistance ratio curves for a thin-layer of strong soil ( $D_{R}=90 \%$ ) embedded within a weak soil $\left(D_{R}=10 \%\right)$ with variation of $H_{t} / B$ from 10 to 50 . Thin-layer effects increase significantly with decreasing layer thickness. When $H_{t}=50$, the thickness is larger than $2 Z_{s}\left(Z_{s} \approx 20\right.$ for the test with $D_{R}=10 \%$ overlying $\left.D_{R}=90 \%\right)$ and the maximum value of $\eta^{\prime}$ reaches 1 , indicating no thin-layer effect.

The effect of the relative density of the strong soil (Fig. 13a) and weak soil (Fig. 13b) on the influence of the thin-layer are investigated with a constant thin-layer thickness $\left(H_{t}=\right.$ $20 B)$. In Fig. 13a, the value of $\eta_{\max }^{\prime}$ seems to decrease linearly $\left(\Delta \eta_{\max }^{\prime} / \Delta D_{R} \approx-0.2 / 20 \%=\right.$ -0.01) when the value of $D_{R}$ of the thin strong soil is increased from $30 \%$ to $90 \%$. In Fig. 13b, the effect of the value of $D_{R}$ of the weak soil is shown to have less of an effect, where $\Delta \eta_{\max }^{\prime} / \Delta D_{R} \approx 0.15 / 20 \%=0.0075$.

The variation of $\eta_{\max }^{\prime}$ with the thickness of the thin-layer is illustrated in Fig. 14. The value of $1-\eta_{\text {max }}^{\prime}$ indicates the magnitude of the thin-layer effect, which vanishes gradually as $H_{t}$ increases. The curves also indicate the effect of varying $D_{R, s}$ and $D_{R, w}$. An increase of $D_{R}$ of the strong soil or a decrease of $D_{R}$ of the weak soil is shown to increase the effects of the thin strong soil layer.

Weak soil within strong soil

Fig. 15 shows the resistance ratio curves for a thin-layer of weak soil ( $D_{R}=10 \%$ ) embedded within a strong soil $\left(D_{R}=90 \%\right)$ with variation of $H_{t} / B$ from 5 to 25 . Compared to the thin layer of strong soil, a smaller size of $H_{t}$ is required to produce a thin-layer effect, owing to the smaller size of the influence zone in the weak soil. When $H_{t}<15$, the minimum resistance ratio starts to be affected by the strong layers. However, the existence of the weak thin-layer 
significantly and extensively affects the measurements in both strong layers. When the thin weak layer has a significant effect in this situation, an estimate of the actual $q_{c, w}$ is required to prevent an over-prediction of soil strength. For example, for the test with $H_{t} / B=5$ in Fig. 15, the measured minimum penetration resistance is about $848.5 \mathrm{kPa}$ for $\eta_{\min }^{\prime}=0.167$, whereas the actual penetration resistance in uniform weak soil is $309.1 k P a$, which is only $36 \%$ of the measured resistance.

The variation of $\eta^{\prime}$ with $D_{R}$ in each soil layer is shown in Fig. 16, with a constant $H_{t}=10 \mathrm{~B}$. Fig. 16a shows that an increase of the thin-layer effect (given by an increase in $\left.\eta_{\min }^{\prime}-0\right)$ is observed when the value of $D_{R}$ of the weak soil is increased. However, as the value of $D_{R}$ of the thin weak layer is increased, the effect of the thin-layer on the penetration resistance within the surrounding strong soil becomes less significant. Inversely, Fig. 16b shows that when the value of $D_{R}$ of the strong soil is increased, the thin-layer effect reduces $\left(\eta_{\min }^{\prime}\right.$ approaches zero) but the effect of the thin weak layer on the penetration resistance in the surrounding strong soil becomes more significant.

Consistent with the gradual reduction of the thin-layer effect with an increase of $H_{t}$ shown for a thin strong layer of soil (Fig. 14), the minimum resistance ratio in the sandwiched weak soil decreases with $H_{t}$, implying a decrease in the thin-layer effect (Fig. 17). A decrease of $D_{R, w}$ or an increase of $D_{R, s}$ are also shown to reduce the thin-layer effect of the embedded weak soil.

\section{Comparisons with field data and numerical results}

For penetration in thin layered soils, most of the research and applications reported from the literature are based on the simplified elastic solution carried out by Vreugdenhil et al. (1994). Robertson and Fear (1995) proposed a correction factor $K_{H}=q_{c, s} / q_{c, \max }$ for the interpretation of penetration in thin sand layers embedded in softer deposits, which is used to estimate the actual properties in thinly interbedded soils based on the measured maximum tip resistance $\left(q_{c, \max }\right)$. The degradation curves of $K_{H}$ with $H_{t}$ were also investigated for different stiffness ratios $G_{s} / G_{w}$ (i.e. $q_{c, s} / q_{c, w}$ ), based on the method of Vreugdenhil et al. 
(1994). After some field data reported by an unpublished work by Robertson and Castro, indicating the over-prediction of the thin-layer effects from the elastic solution, Youd et al. (2001) plotted this area with field data, and provided an empirical equation of $K_{H}$ for the lower bound of the field observation.

A series of numerical simulations was also carried out by Ahmadi and Robertson (2005) to examine the variation of the correction factor $K_{H}$ with thickness $H_{t}$, following the simulation of two-layered tests mentioned in the previous section. The sample was a thin sand layer embedded in soft clay layers under a relatively low confining stress $\left(\sigma_{v 0}^{\prime}=70 \mathrm{kPa}, \sigma_{h 0}^{\prime}=\right.$ $35 \mathrm{kPa})$. Loose sand $\left(D_{R, s}=30 \%\right)$, medium dense sand $\left(D_{R, s}=50 \%\right)$, and dense sand $\left(D_{R, s}=90 \%\right)$ were investigated.

Fig. 18 compares the parameters $\left(K_{H}\right.$ and $\left.\eta_{\max }^{\prime}\right)$ for investigation of the thin-layer effects. Again, the soil properties for the comparisons are equivalent to those from the simulations of Ahmadi and Robertson (2005). The value of $K_{H}$ in Fig. 18a decreases to 1 when the layer thickness is increased (i.e. $K_{H}=1$ implies no thin-layer effects). The field data provided by Robertson and Castro for the NCEER workshop (as reported by Youd et al. 2001) is shown in the shaded area. The current analytical solution for the equivalent problem provides the magnitude of $\eta_{\text {max }}^{\prime}$, and therefore calculates $K_{H}=\frac{q_{s} / q_{w}}{\left(q_{s} / q_{w}-1\right) \eta_{\max }^{\prime}+1}$.

Comparing with the field data, the analytical results obtained using the cavity expansion analysis proposed in this paper show similar trends of $K_{H}$, and illustrate the effect of the relative soil properties. The results from this analysis show that for a given thin layer thickness, a stronger thin layer soil has a larger correction factor $K_{H}$. Unfortunately, details of the soil from the field data are not available so it is not possible to make a direct quantitative comparison. The analytical results agree reasonably well with the results of the numerical simulations from Ahmadi and Robertson (2005) (also shown in Fig. 18a), for the same assumed ground conditions.

Fig. 18b compares values of $\eta_{\max }^{\prime}$ obtained using the proposed cavity expansion method with the numerical results and results obtained using the elastic solutions of Vreugdenhil 
et al. (1994). The numerical results are based on the data in Fig. 18a and the transition curves in Ahmadi and Robertson (2005). The data illustrate the effect of layer thickness $\left(H_{t}\right)$ and relative density of the thin layer, and although similar trends are noted, the elastic solution is shown to predict much higher thin-layer effects (given by $1-\eta_{\max }^{\prime}$ ) than the numerical predictions. The current analytical elastic-plastic solutions provide a more reasonable evaluation of the thin-layer effects, which show better agreement with the numerical results.

\section{DISCUSSION}

It should be noted that the values of the many parameters $\left(Z_{s}\right.$ and $Z_{w} ; K_{H}$ and $\left.\eta_{\max }^{\prime}\right)$ were calculated for specific situations and should not be taken as generally applicable. The influence zones depend not only on the soil properties and profiles, but also on the stress state and probe diameter, which are included in the analytical calculations. The magnitude of in situ confining stress has an impact on the size of the influence zones. A higher stress condition is found to result in smaller values of $Z_{s}$ and $Z_{w}$, though the impact was found to be relatively small. All of the results with distance to the interface have been normalized by the probe diameter. The size of the influence zones are proportional to the probe diameter, and thus a smaller penetrometer will have a less significant layered effect and will be better at detecting thin layers, as mentioned in Ahmadi and Robertson (2005) and Xu and Lehane (2008). Similarly, the thin-layer effects are also influenced by stress condition and probe diameter.

Various complexities that affect penetrometer response were intentionally disregarded from the analysis presented in the paper so that the intended focus (i.e. layering effects) would not be diminished. For example, the cone and shaft interface friction (Lee 1990), stress gradient (Bolton et al. 1999), and the pore-water pressure dissipation of the surrounding soil (Sultan and Lafuerza 2013) influence penetrometer readings. The purpose of this paper was to illustrate how an analytical cavity expansion methodology could be applied to the analysis of layered soils. The proposed method could be modified to account for additional 
complexities, or even to the application of other penetration problems such as the ball penetration test. For example, it may be possible to modify the analysis to consider a stress gradient by including many layers of soil with parameters changing to account for increasing stress level, and the ground surface modelled as an extremely weak soil layer.

The analytical solutions presented here used the mean stress as the in situ hydrostatic stress. The effect of the coefficient of at-rest earth pressure $\left(K_{0}\right)$ was not considered. Further work on the application to layered scenarios could use cylindrical solutions with non-isotropic in situ stress condition (e.g. Chen and Abousleiman 2013), or focus on developing elliptical cavity expansion solutions (e.g. Kong et al. 2014).

\section{CONCLUSIONS}

Analytical cavity expansion solutions in two concentrically layered soils were applied to the interpretation of CPT results, with specific focus on the layered effects during penetration. A discussion on concentric and horizontal layering was provided to validate the relevance between the two types of models. The analogy between the CPT and cavity expansion in two-layered soils was described, and a combination approach for predicting tip resistance in two-layered soils was provided. The analyses of CPT in two-layered soils highlighted the effect of respective soil properties (strength, stiffness) on CPT measurements within the influence zones around the two-soil interface. The resistance ratios and influence zones in the weak and strong soils were found to be affected by the soil properties of both layers. The results provided good comparisons with numerical results and the elastic solutions. A simple superposition method of the two-layered analytical results was applied for the analysis of penetration in multi-layered soils. The thin-layer effects were investigated by analyzing a thin layer of both strong and weak soils. The correction factor $\left(K_{H}\right)$ determined using the proposed solution compared well with field data and numerical results, and the proposed parameters $\left(\eta_{\max }^{\prime}, \eta_{\text {min }}^{\prime}\right)$ were shown to give a good measurement of the thin layer effects. The influence of soil properties in each layer as well as layer geometry on the magnitude of thin-layer effect was demonstrated. It was also shown that the results of $\eta_{\text {max }}^{\prime}$ showed better 

agreement with numerical predictions than those obtained using existing elastic solutions. 
The following symbols are used in this paper:

$a_{0}, a=$ radii of cavity;

$b_{0}, b=$ radii of Soil A/B interface;

$q_{c}=$ cone tip resistance;

$s_{u}=$ undrained shear strength for clay;

$C=$ cohesion of soil;

$B=$ diameter of probe or pile;

$D=$ diameter of centrifuge container;

$D_{R}=$ relative density of soil;

$E=$ Young's modulus of soil;

$G, G_{0}=$ shear modulus and small-strain shear modulus of soil;

$G_{s}=$ specific gravity;

$H=$ distance to soil interface;

430

$H_{t}=$ thickness of sandwiched soil layer;

$K_{0}=$ coefficient of at-rest earth pressure;

$K_{H}=$ correction factor for thin-layer effects;

$P_{0}=$ initial cavity pressure and in situ hydrostatic stress;

$P_{a}=$ radial stress at the cavity;

$P_{\text {lim }}=$ limit pressure of cavity expansion;

$R=$ radius of probe or pile;

$Z_{s}, Z_{w}=$ size of influence zones in strong and weal soils;

$\phi=$ friction angle of soil;

$\psi=$ dilation angle of soil;

$\eta^{\prime}=$ cone tip resistance ratio in layered soils;

$\nu=$ Poisson's ratio of soil; and

$\sigma_{a t m}=$ atmospheric pressure, as the reference pressure. 


\section{APPENDIX 1. APPROACH FOR PREDICTION OF SOIL MODEL PARAMETERS}

The analytical cavity expansion solutions use a non-associated Mohr-Coulomb soil model, where five parameters are required to represent the soil stress-strain relationship: Young's modulus $(E)$, Poisson's ratio $(\nu)$, friction angle $(\phi)$, cohesion $(C)$ and dilation angle $(\psi)$. This appendix provides a simple approach to determine the soil model parameters of Fraction E silica sand.

Many analytical models have been proposed to predict the stress-strain behaviour for granular material (e.g. Santamarina and Cascante 1996, Liao et al. 2000, McDowell and Bolton 2001), especially for the evaluation of small-strain shear modulus, $G_{0}$ (where $G=$ $E /[2(1+\nu)])$. The Fahey-Carter model (Fahey and Carter 1993) was used in this work to deifine $G_{0}$. For non-linear elastic behaviour, $G_{0}$ is defined as a function of in situ confining stress $\left(P_{0}\right)$, as follow:

$$
\frac{G_{0}}{\sigma_{a t m}}=c^{\prime}\left(\frac{P_{0}}{\sigma_{a t m}}\right)^{n^{\prime}}
$$

where $c^{\prime}$ and $n^{\prime}$ are soil-specific parameters, and $\sigma_{a t m}$ is atmospheric pressure.

Shear stiffness degradation with increasing shear strain is not included in the analytical solutions, hence $G_{0}$ is used to represent the shear stiffness of the soil. Note that, due to the model used for $G_{0}$, the estimated shear modulus is independent of soil density. A different model for $G_{0}$ could be adopted to consider the effect of soil density. Poisson's ratio is defined as 0.2 , which is reasonable for many soils (Mitchell and Soga 2005). The soil properties are based on Fraction E silica sand, with data obtained from Tan (1990) and Zhao (2008). This same sand was also used in the centrifuge tests reported in Mo et al. (2015). Curvefitting using the Fahey-Cater model resulted in the soil-specific parameters of $c^{\prime}=1000$ and $n^{\prime}=0.5$.

In terms of strength and dilatancy of sands, Bolton (1986) proposed a correlation between peak friction angle $\left(\phi_{\max }^{\prime}\right)$, critical state friction angle $\left(\phi_{\text {crit }}^{\prime}\right)$ and peak dilatancy $\left(\psi_{\max }\right)$, and introduced a relative dilatancy index $\left(I_{R}\right)$, based on triaxial tests of 17 sands: $\phi_{\max }^{\prime}-\phi_{c r i t}^{\prime}=$ $0.8 \psi_{\max }=3 I_{R}$ (the triaxial scenario was used according to the assumption of spherical 
cavity expansion). $I_{R}$ was also defined as a function of relative density $\left(D_{R}\right)$ and in situ confining stress $\left(P_{0}\right): I_{R}=D_{R}\left(Q^{\prime}-\ln P_{0}\right)-R^{\prime}$, where $Q^{\prime}$ and $R^{\prime}$ are material constants; $D_{R}$ is the relative density value in ' $\%$ ' and $P_{0}$ is in $k P a$.

For Leighton Buzzard sand, these material constants were obtained from triaxial tests by Wang (2005): $Q^{\prime}=9.4$ and $R^{\prime}=0.28$. In addition, the cohesion $(C)$ was set as zero for the cohesionless soil. Considering the assumption of constant material parameters for the analytical solution, a simple averaging method suggested by Randolph et al. (1994) was used for soil between the initial and critical state: $\phi=\frac{\phi_{\text {max }}^{\prime}+\phi_{c r i t}^{\prime}}{2}$ and $\psi=\frac{\psi_{\max }}{2}$.

\section{REFERENCES}

Ahmadi, M. M., Byrne, P. M., and Campanella, R. G. (2005). "Cone tip resistance in sand: modeling, verification, and applications." Canadian Geotechnical Journal, 42(4), 977-993.

Ahmadi, M. M. and Robertson, P. K. (2005). "Thin-layer effects on the CPT q(c) measurement." Canadian Geotechnical Journal, 42(5), 1302-1317.

Bishop, R. F., Hill, R., and Mott, N. F. (1945). "The theory of indentation and hardness tests." Proceedings of the Physical Society of London, 57(321), 147-159.

Bolton, M. D. (1986). "The strength and dilatancy of sands." Géotechnique, 36(1), 65-78.

Bolton, M. D., Gui, M. W., Garnier, J., Corte, J. F., Bagge, G., Laue, J., and Renzi, R. (1999). "Centrifuge cone penetration tests in sand." Géotechnique, 49(4), 543-552.

Chen, S. L. and Abousleiman, Y. N. (2013). "Exact drained solution for cylindrical cavity expansion in modified cam clay soil." Géotechnique, 63(6), 510-517.

Fahey, M. and Carter, J. P. (1993). "A finite-element study of the pressuremeter test in sand using a nonlinear elastic-plastic model." Canadian Geotechnical Journal, 30(2), 348-362.

Gibson, R. and Anderson, W. (1961). "In situ measurement of soil properties with the pressuremeter." Civil Engineering and Public Works Review, 56(658), 615-618.

Hird, C. C., Johnson, P., and Sills, G. C. (2003). "Performance of miniature piezocones in thinly layered soils." Géotechnique, 53(10), 885-900.

Kong, G. Q., Zhou, H., Cao, Z. H., and Liu, H. L. (2014). "Analytical solution for pressure- 
controlled elliptical cavity expansion in elasticperfectly plastic soil." Géotechnique Letters, $4,72-78$.

Ladanyi, B. and Johnston, G. H. (1974). "Behaviour of circular footings and plate anchors embedded in permafrost." Canadian Geotechnical Journal, 11, 531-553.

Lee, S. Y. (1990). "Centrifuge modelling of cone penetration testing in cohesionless soils." Ph.D. thesis, University of Cambridge, UK.

Liao, C. L., Chan, T. C., Suiker, A. S. J., and Chang, C. S. (2000). "Pressure-dependent elastic moduli of granular assemblies." International Journal for Numerical and Analytical Methods in Geomechanics, 24(3), 265-279.

McDowell, G. and Bolton, M. D. (2001). "Micro mechanics of elastic soil." Soils and Foundations, 41(6), 147-152.

Meyerhof, G. G. (1976). "Bearing capacity and settlement of pile foundations." Journal of the Geotechnical Engineering Division-ASCE, 102(3), 195-228.

Meyerhof, G. G. (1977). "Bearing capacity of piles in layered soils." Proceedings of the 8th International Conference on Soil Mechanics and Foundation Engineering, 645-650.

Meyerhof, G. G. (1983). "Scale effects of ultimate pile capacity." Journal of Geotechnical Engineering-ASCE, 109(6), 797-806.

Meyerhof, G. G. and Sastry, V. V. R. N. (1978a). "Bearing capacity of piles in layered soils.1. clay overlying sand." Canadian Geotechnical Journal, 15(2), 171-182.

Meyerhof, G. G. and Sastry, V. V. R. N. (1978b). "Bearing capacity of piles in layered soils.2. sand overlying clay." Canadian Geotechnical Journal, 15(2), 183-189.

Mitchell, J. K. and Soga, K. (2005). Fundamentals of soil behaviour. John Wiley and Sons, 3rd edition.

Mo, P. Q., Marshall, A. M., and Yu, H. S. (2013). "Centrifuge modelling of CPT in layered soils." Geotechnical and Geophysical Site Characterization 4 - Proceedings of the 4th International Conference on Site Characterization 4, ISC-4, Vol. 1, 219-225.

Mo, P. Q., Marshall, A. M., and Yu, H. S. (2014). "Elastic-plastic solutions for expanding 
cavities embedded in two different cohesive-frictional materials." International Journal for Numerical and Analytical Methods in Geomechanics, 38(9), 961-977.

Mo, P. Q., Marshall, A. M., and Yu, H. S. (2015). "Centrifuge modelling of cone penetration tests in layered soils." Géotechnique, 65(6), 468-481.

Randolph, M. F., Dolwin, J., and Beck, R. (1994). "Design of driven piles in sand." Géotechnique, 44(3), 427-448.

Robertson, P. K. and Fear, C. E. (1995). "Liquefaction of sands and its evaluation." Proceedings of the 1st International Conference on Earthquake Geotechnical Engineering, Vol. 3, $1253-1289$

Salgado, R., Mitchell, J. K., and Jamiolkowski, M. (1997). "Cavity expansion and penetration resistance in sand." Journal of Geotechnical and Geoenvironmental Engineering, 123(4), $344-354$.

Santamarina, J. C. and Cascante, G. (1996). "Stress anisotropy and wave propagation: A micromechanical view." Canadian Geotechnical Journal, 33(5), 770-782.

Sayed, S. M. and Hamed, M. A. (1987). "Expansion of cavities in layered elastic system." International Journal for Numerical and Analytical Methods in Geomechanics, 11(2), 203213.

Sultan, N. and Lafuerza, S. (2013). "In situ equilibrium pore-water pressures derived from partial piezoprobe dissipation tests in marine sediments." Canadian Geotechnical Journal, 50(12), 1294-1305.

Tan, F. S. C. (1990). "Centrifuge and theoretical modelling of conical footings on sand." Ph.D. thesis, University of Cambridge, UK.

van den Berg, P. (1994). "Analysis of soil penetration." Ph.D. thesis, Delft University of Technology, Delft University of Technology.

Vesic, A. S. (1977). "Design of pile foundations." National Cooperation Highway Research Program, Synthesis of Highway Practice 42.

Vreugdenhil, R., Davis, R., and Berrill, J. (1994). "Interpretation of cone penetration results 
in multilayered soils." International Journal for Numerical and Analytical Methods in Geomechanics, 18(9), 585-599.

Walker, J. and Yu, H. S. (2010). "Analysis of the cone penetration test in layered clay." Géotechnique, 60(12), 939-948.

Wang, J. (2005). "The stress-strain and strength characteristics of portaway sand." Ph.D. thesis, University of Nottingham, UK.

$\mathrm{Xu}, \mathrm{X}$. T. and Lehane, B. M. (2008). "Pile and penetrometer end bearing resistance in two-layered soil profiles." Géotechnique, 58(3), 187-197.

Yasufuku, N. and Hyde, A. F. L. (1995). "Pile end-bearing capacity in crushable sands." Géotechnique, 45(4), 663-676.

Youd, T. L., Idriss, I. M., Andrus, R. D., Arango, I. Castro, G., Chistian, J. T., Dobry, R., Finn, W. D. L., Harder, L. F. J., Hynes, M. E., Ishihara, K., Koester, J. P., Liao, S. S. C., Marcuson, W. F. I., Martin, G. R., Mitchell, J. K., Moriwaki, Y., Power, M. S., Rovertson, P. K., Seed, R. B., and Stokoe, K. H. I. (2001). "Liquefaction resistance of soils: Summary report from the 1996 NCEER and 1998 NCEER/NSF workshops on evaluation of liquefaction resistance of soils." Journal of Geotechnical and Geoenvironmental Engineering, 127(10), 817-833.

Yu, H. S. (2000). Cavity expansion methods in geomechanics. Kluwer Academic Publishers.

Yu, H. S. (2006). "The First James K. Mitchell Lecture In situ soil testing: from mechanics to interpretation." Geomechanics and Geoengineering: An International Journal, 1(3), $165-195$.

Yu, H. S. and Mitchell, J. K. (1998). "Analysis of cone resistance: Review of methods." Journal of Geotechnical and Geoenvironmental Engineering, 124(2), 140-149.

Zhao, Y. (2008). "In situ soil testing for foundation performance prediction." Ph.D. thesis, University of Cambridge, UK. 


\section{${ }_{564} \quad$ List of Tables}

1 Soil model parameters and estimated cone resistance in uniform soil layer . . 
TABLE 1. Soil model parameters and estimated cone resistance in uniform soil layer

\begin{tabular}{ccccccc}
\hline Relative density & \multicolumn{5}{c}{ Soil parameters } & Cone tip resistance \\
\cline { 2 - 5 }$D_{R}(\%)$ & $G(M P a)$ & $\nu$ & $C(k P a)$ & $\phi\left(^{\circ}\right)$ & $\psi\left({ }^{\circ}\right)$ & $q_{c}(k P a)$ \\
\hline 10 & 10.1 & 0.2 & 0 & 33.0 & 1.2 & 309 \\
30 & 10.1 & 0.2 & 0 & 35.8 & 4.8 & 573 \\
50 & 10.1 & 0.2 & 0 & 38.6 & 8.3 & 1064 \\
70 & 10.1 & 0.2 & 0 & 41.5 & 11.8 & 1958 \\
90 & 10.1 & 0.2 & 0 & 44.3 & 15.3 & 3542 \\
\hline
\end{tabular}




\section{List of Figures}

1 Comparison of cavity expansion in concentric regions and cone penetration in horizontal layers (adapted from S. M. Sayed and M. A. Hamed, 1987, "Expansion of cavities in layered elastic system." International Journal Journal for Numerical and Analytical Methods in Geomechanics, Vol. 11, No. 1, pp. 203-213, (c)1999-2016 John Wiley \& Sons, Inc. Reproduced with permission)

2 Numerical models for cavity expansion in: (a) concentric layers; and (b) horizontal layers . . . . . . . . . . . . . . . . . . . . . . 30

3 Cavity pressure with variation of $b_{0} / a_{0}$ in concentric and horizontal layered model when $a / a_{0}=1.2 \ldots \ldots \ldots$. . . . . . . . . . . . . . 31

4 Schematic of cone penetration and cavity expansion in two-layered soils . . .

5 Schematic of combination method for cavity pressure transition in two-layered

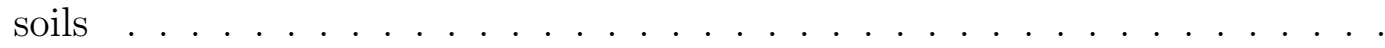

$6 \quad$ Cone tip resistance in two-layered $\left(D_{R}\right)$ soils: (a) variation of weaker soil; (b) variation of stronger soil . . . . . . . . . . . . . . . . . . . . 34

7 Cone tip resistance ratio curves in two-layered $\left(D_{R}\right)$ soils: (a) variation of weaker soil; (b) variation of stronger soil . . . . . . . . . . . . . . . . 35

8 Influence zones in both weak and strong soils with variation of $D_{R} \ldots \ldots$

9 Comparison of cone tip resistance ratio $\left(\eta^{\prime}\right)$ in two-layered soils: (a) loose sand over dense sand; (b) soft clay over dense sand . . . . . . . . . . . . . . 37

10 Schematic of cone penetration in multi-layered soils: strong soil embedded in weak soils . . . . . . . . . . . . . . . . . . . . . . . . . . . . 38

11 Schematic of cone tip resistance ratio $\left(\eta^{\prime}\right)$ in thin-layered soils: (a) strong soil embedded in weak soils, and (b) weak soil embedded in strong soils . . . . .

12 Resistance ratio curves for thin-layer of strong soil $\left(D_{R}=90 \%\right)$ sandwiched by soils with $D_{R}=10 \%$, with variation of $H_{t} / B$ from 10 to $50 \ldots$. . . . 
13 Resistance ratio curves for thin-layer of strong soil $\left(H_{t} / B=20\right)$ : (a) varying $D_{R}$ in strong soil; $(\mathrm{b})$ varying $D_{R}$ in weak soil . . . . . . . . . . . . . 41

14 Strong soil within weak soil: variation of the maximum resistance ratio $\eta_{\max }^{\prime}$ with the thickness of the thin-layer: (a) varying $D_{R}$ in strong soil; (b) varying $D_{R}$ in weak soil . . . . . . . . . . . . . . .

15 Resistance ratio curves for thin-layer of weak soil $\left(D_{R}=10 \%\right)$ sandwiched by soils with $D_{R}=90 \%$ ), with variation of $H_{t} / B$ from 5 to $25 \ldots . . . . .43$

16 Resistance ratio curves for thin-layer of weak soil $\left(H_{t} / B=10\right)$ : (a) varying $D_{R}$ in weak soil; (b) varying $D_{R}$ in strong soil . . . . . . . . . . . . . . . . 44

17 Variation of the minimum resistance ratio $\eta_{\min }^{\prime}$ with the thickness of the thinlayer: (a) varying $D_{R}$ in weak soil; (b) varying $D_{R}$ in strong soil . . . . . . 45

18 Comparisons of the parameters for investigation of thin-layer effects: (a) $K_{H}$;

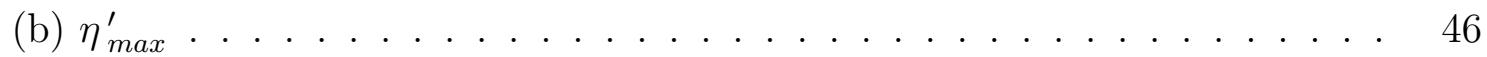



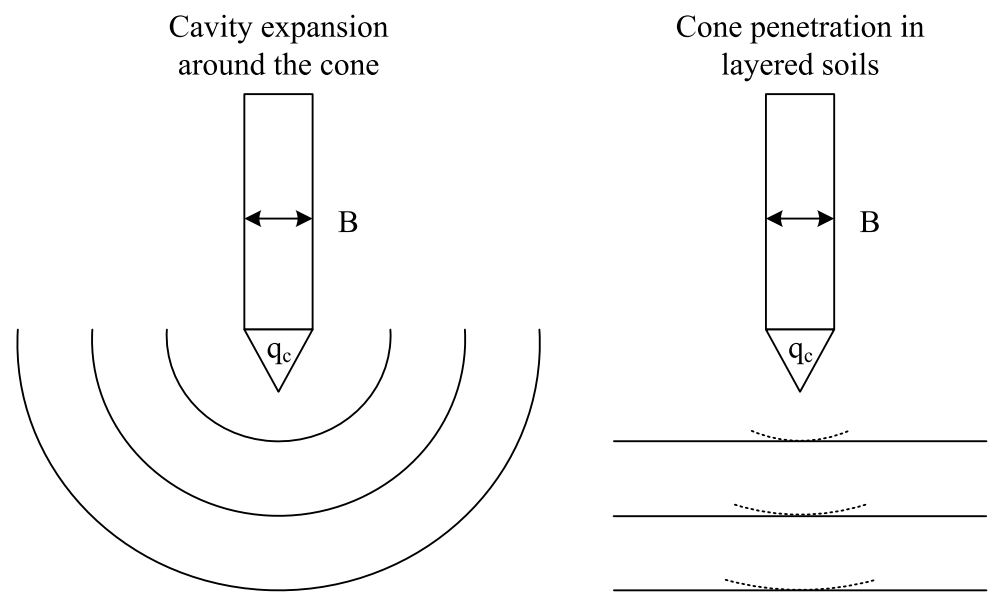

FIG. 1. Comparison of cavity expansion in concentric regions and cone penetration in horizontal layers (adapted from S. M. Sayed and M. A. Hamed, 1987, "Expansion of cavities in layered elastic system." International Journal Journal for Numerical and Analytical Methods in Geomechanics, Vol. 11, No. 1, pp. $203-213$, (c) $1999-2016$ John Wiley \& Sons, Inc. Reproduced with permission) 
FIG. 2. Numerical models for cavity expansion in: (a) concentric layers; and (b) horizontal layers 


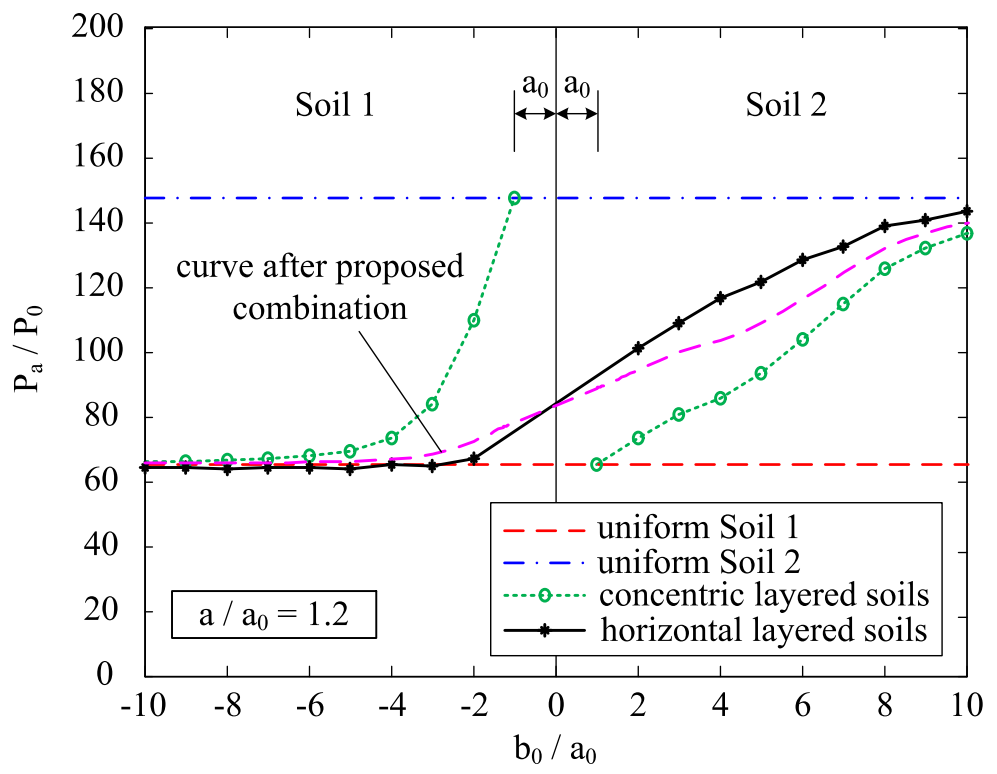

FIG. 3. Cavity pressure with variation of $b_{0} / a_{0}$ in concentric and horizontal layered model when $a / a_{0}=1.2$ 


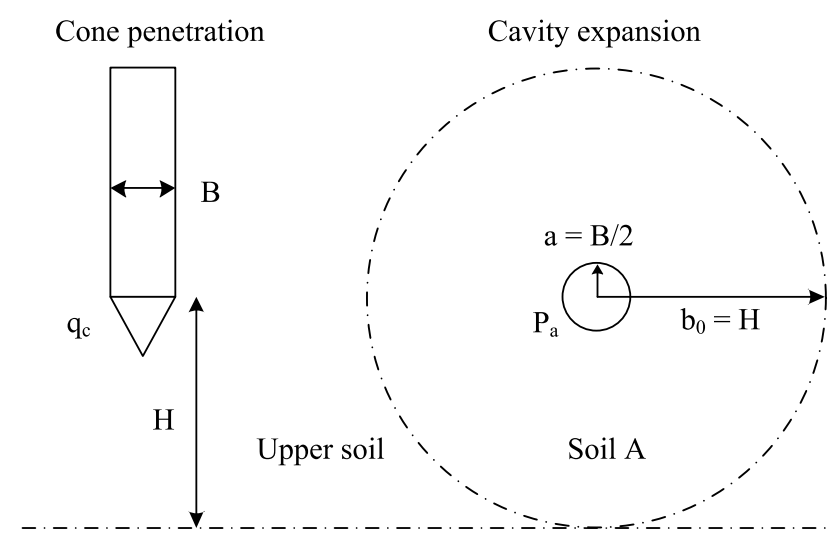

Lower soil

Soil B

FIG. 4. Schematic of cone penetration and cavity expansion in two-layered soils 
FIG. 5. Schematic of combination method for cavity pressure transition in two-layered soils 


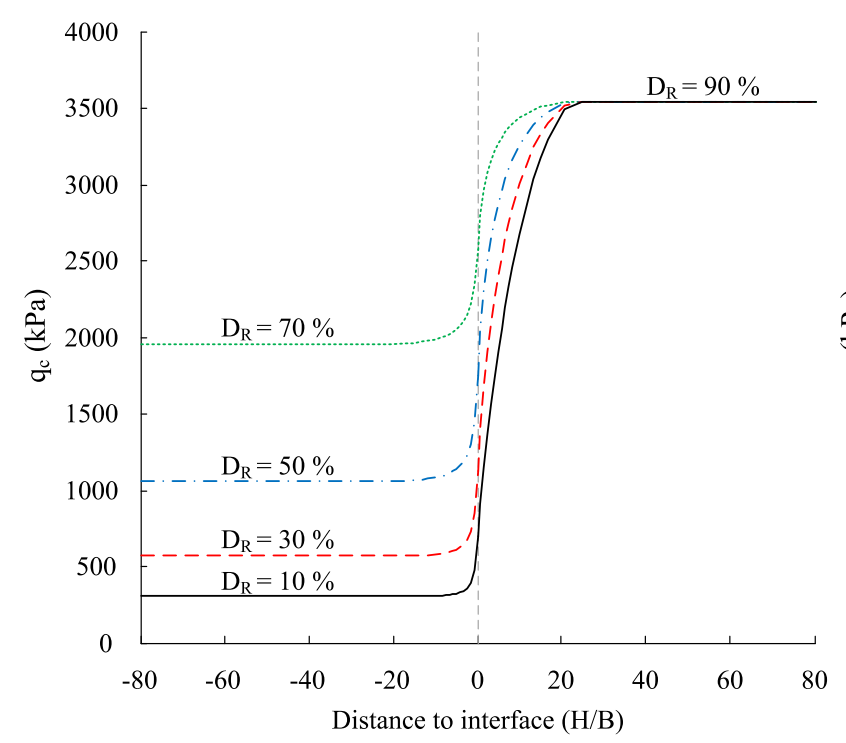

(a)

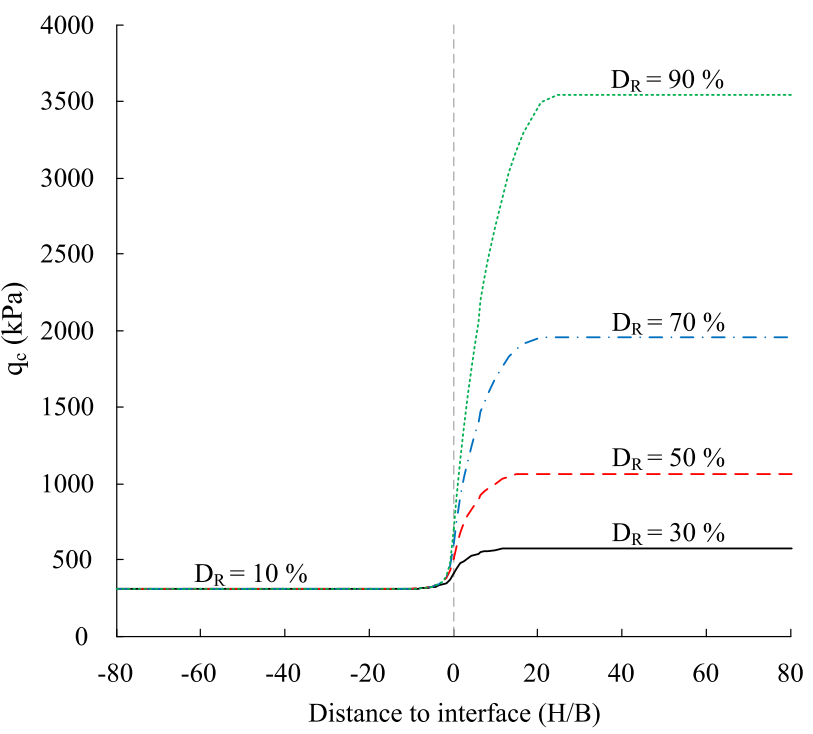

(b)

FIG. 6. Cone tip resistance in two-layered $\left(D_{R}\right)$ soils: (a) variation of weaker soil; (b) variation of stronger soil 


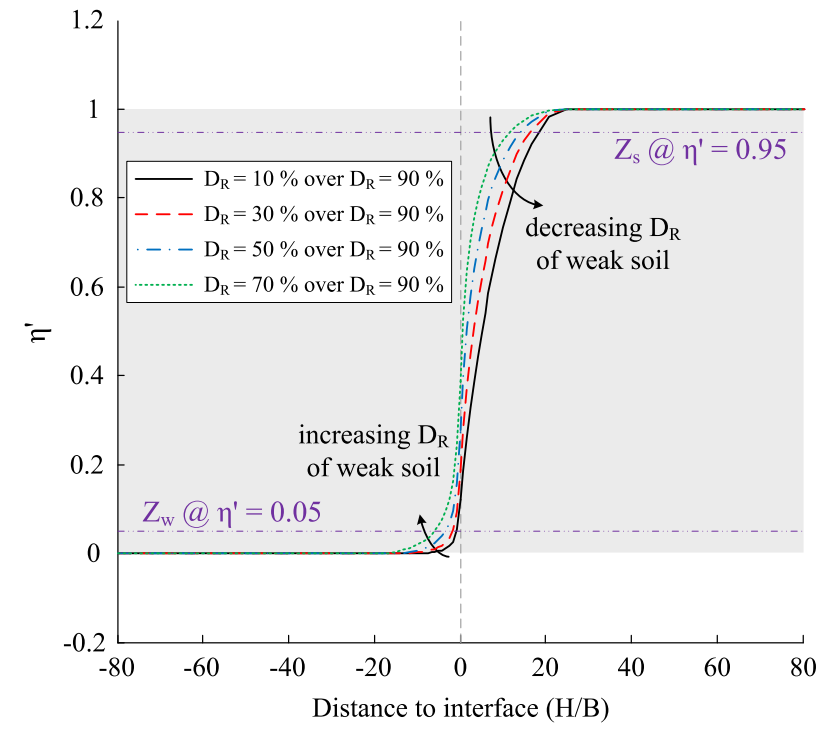

(a)

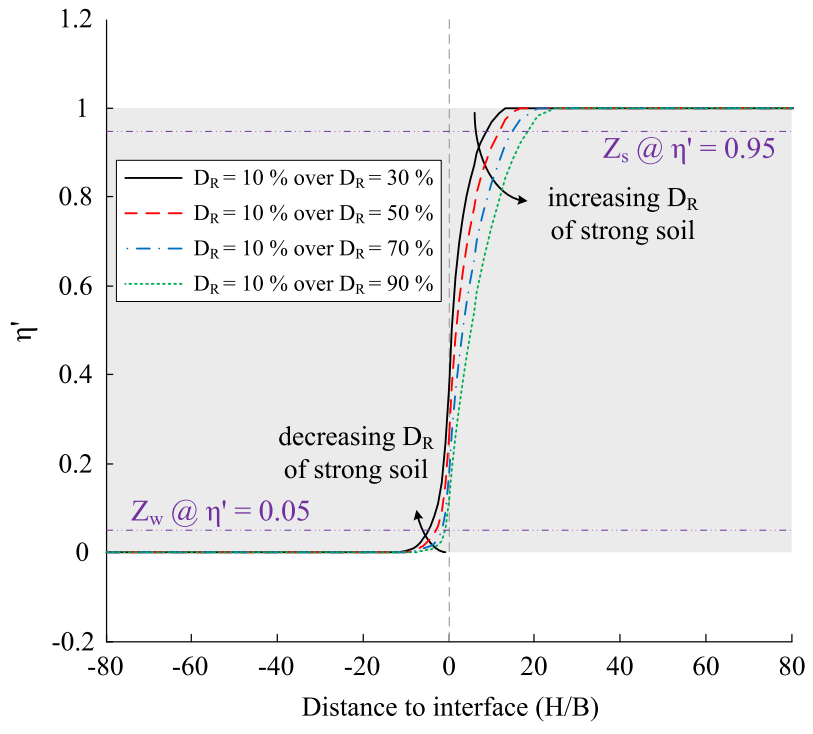

(b)

FIG. 7. Cone tip resistance ratio curves in two-layered $\left(D_{R}\right)$ soils: (a) variation of weaker soil; (b) variation of stronger soil 


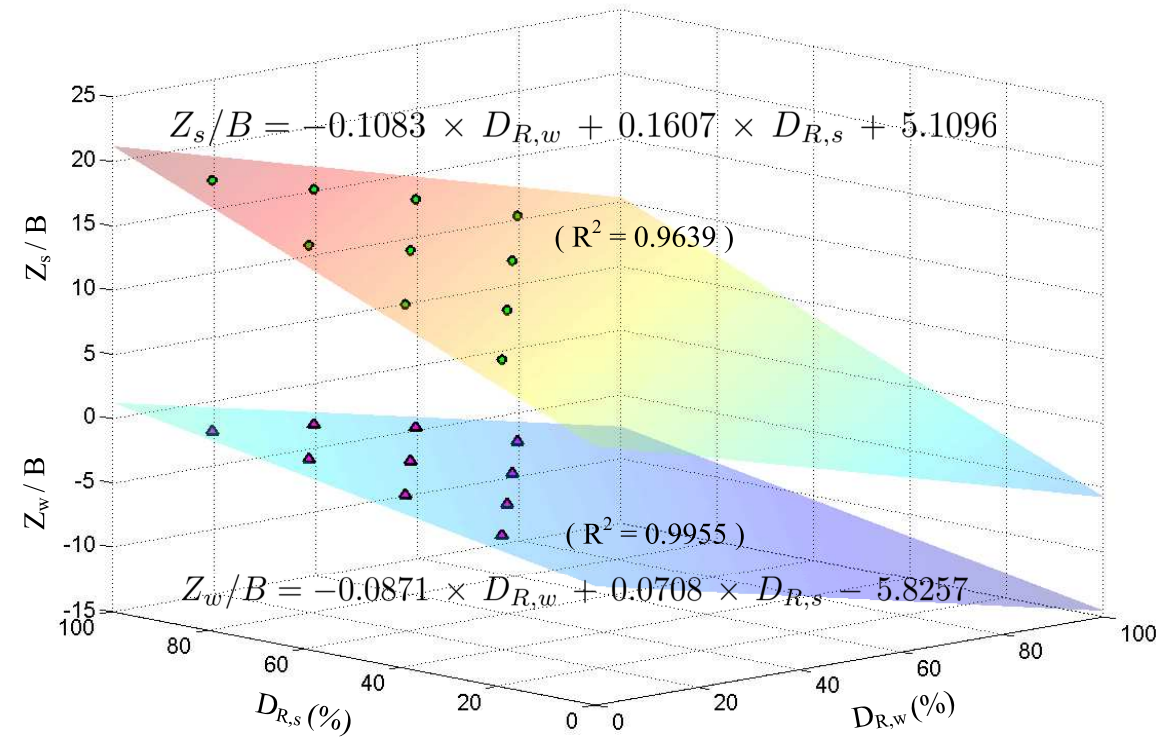

FIG. 8. Influence zones in both weak and strong soils with variation of $D_{R}$ 


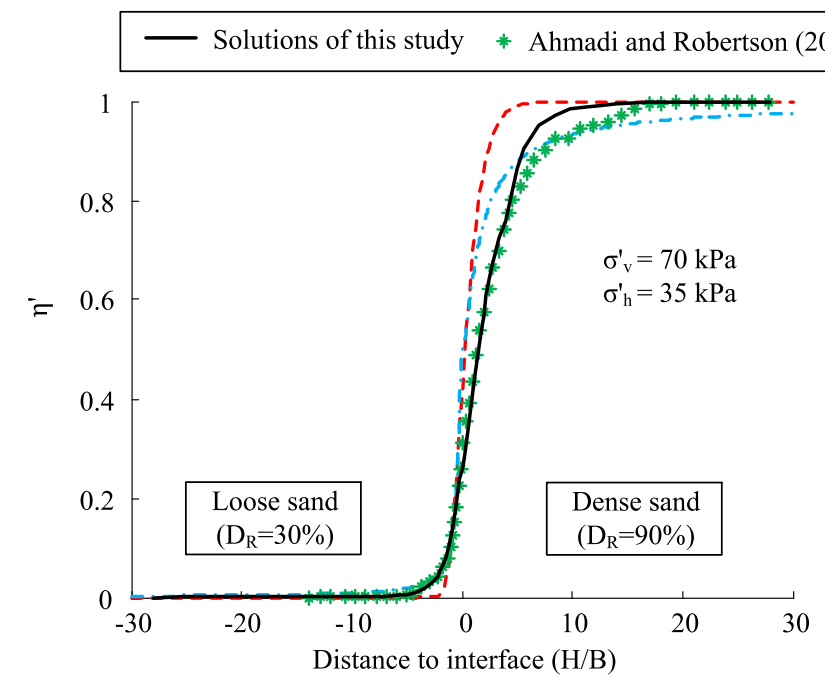

( a)

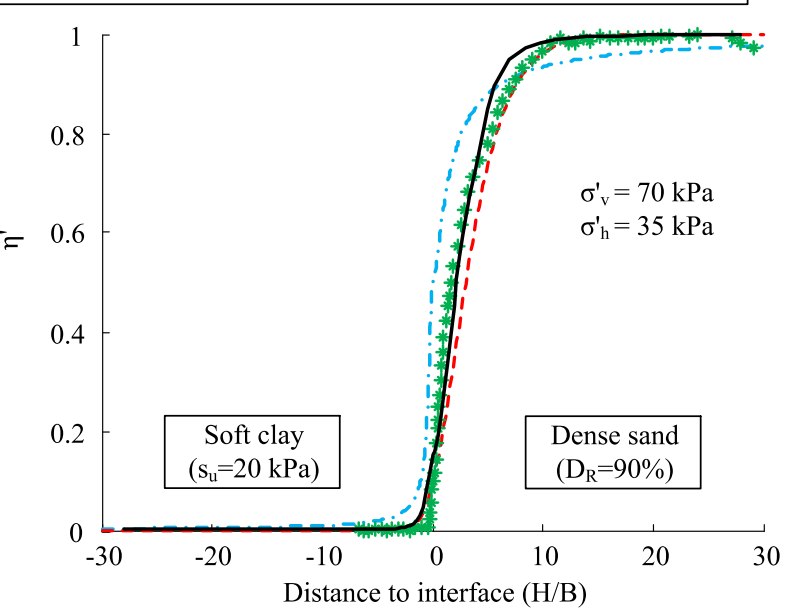

( b )

FIG. 9. Comparison of cone tip resistance ratio $\left(\eta^{\prime}\right)$ in two-layered soils: (a) loose sand over dense sand; (b) soft clay over dense sand 


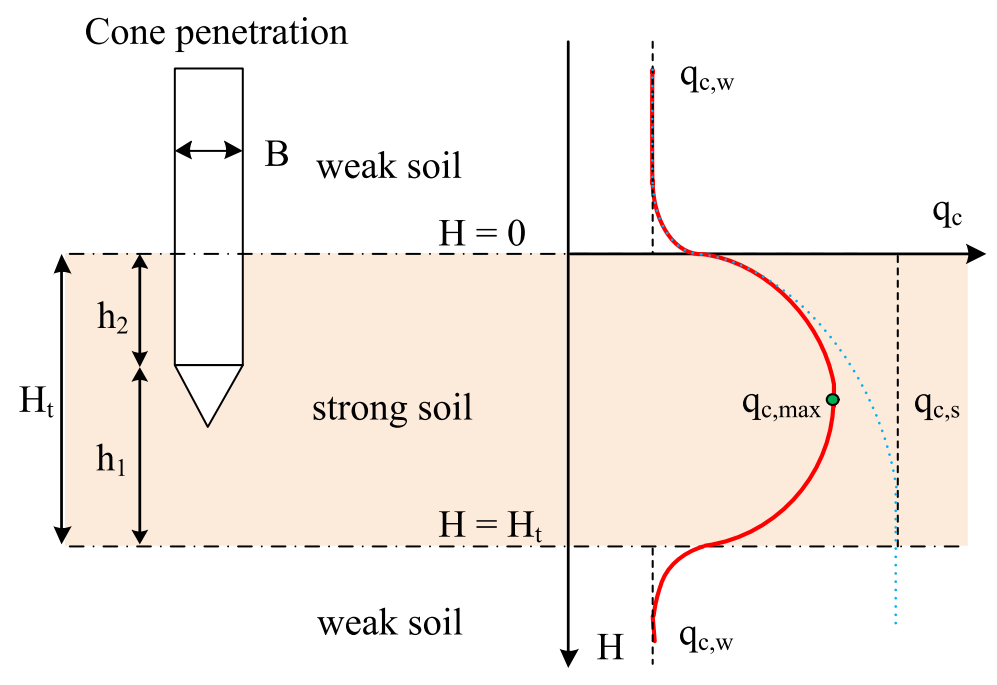

FIG. 10. Schematic of cone penetration in multi-layered soils: strong soil embedded in weak soils 


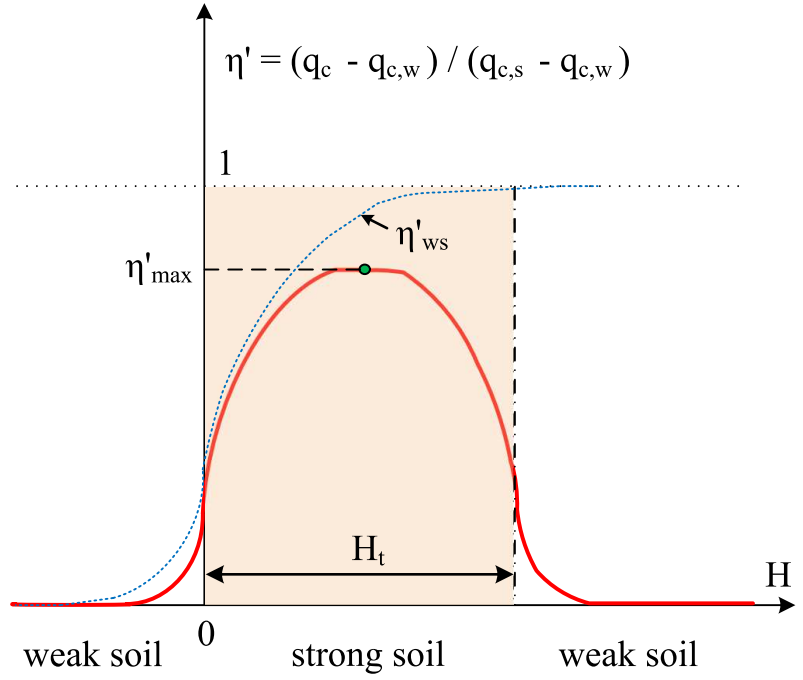

(a)

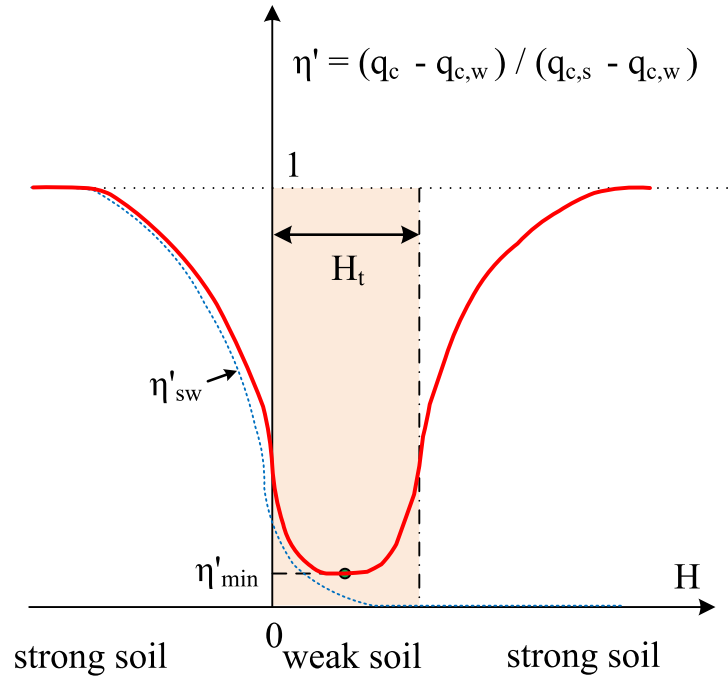

(b)

FIG. 11. Schematic of cone tip resistance ratio $\left(\eta^{\prime}\right)$ in thin-layered soils: (a) strong soil embedded in weak soils, and (b) weak soil embedded in strong soils 
FIG. 12. Resistance ratio curves for thin-layer of strong soil $\left(D_{R}=90 \%\right)$ sandwiched by soils with $D_{R}=10 \%$, with variation of $H_{t} / B$ from 10 to 50 


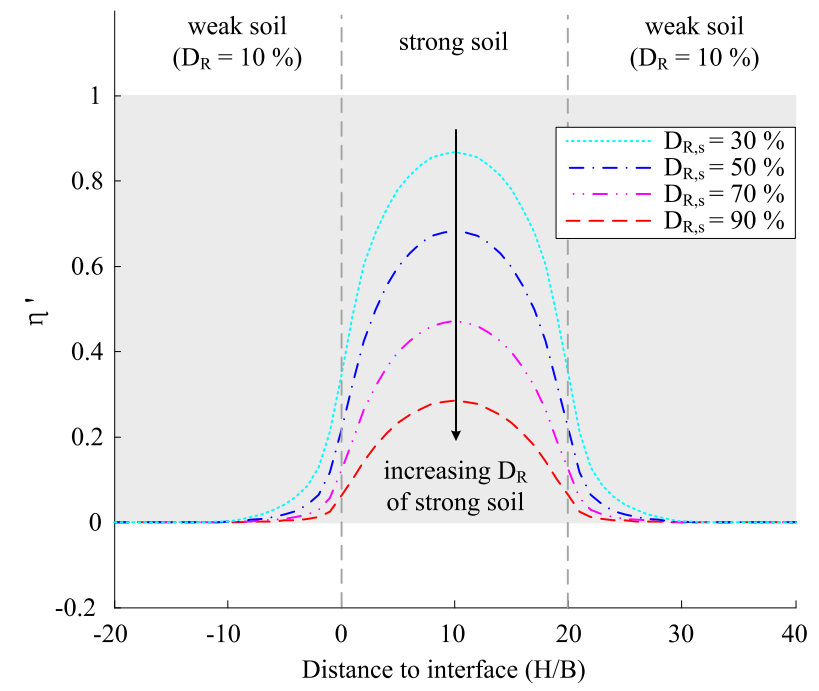

(a)

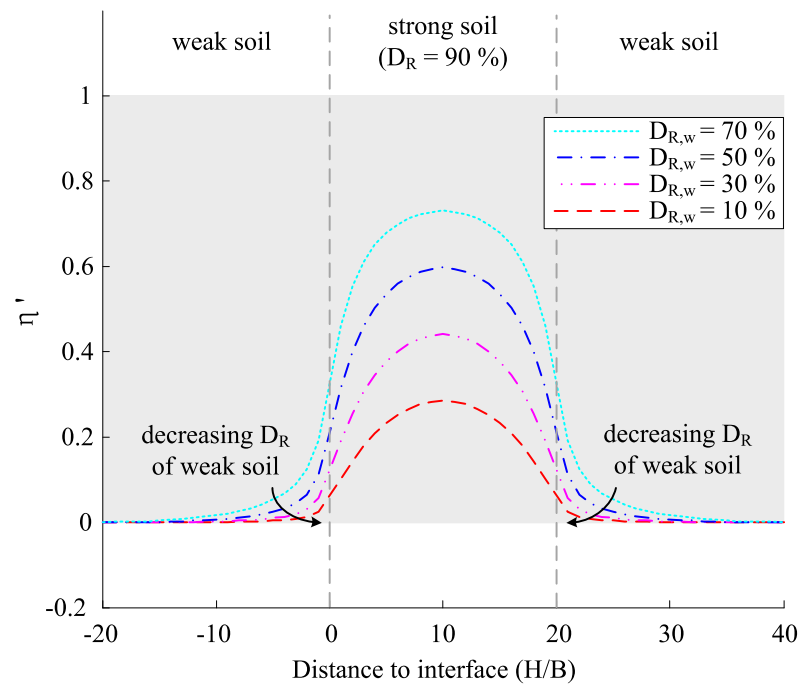

(b)

FIG. 13. Resistance ratio curves for thin-layer of strong soil $\left(H_{t} / B=20\right)$ : (a) varying $D_{R}$ in strong soil; (b) varying $D_{R}$ in weak soil 


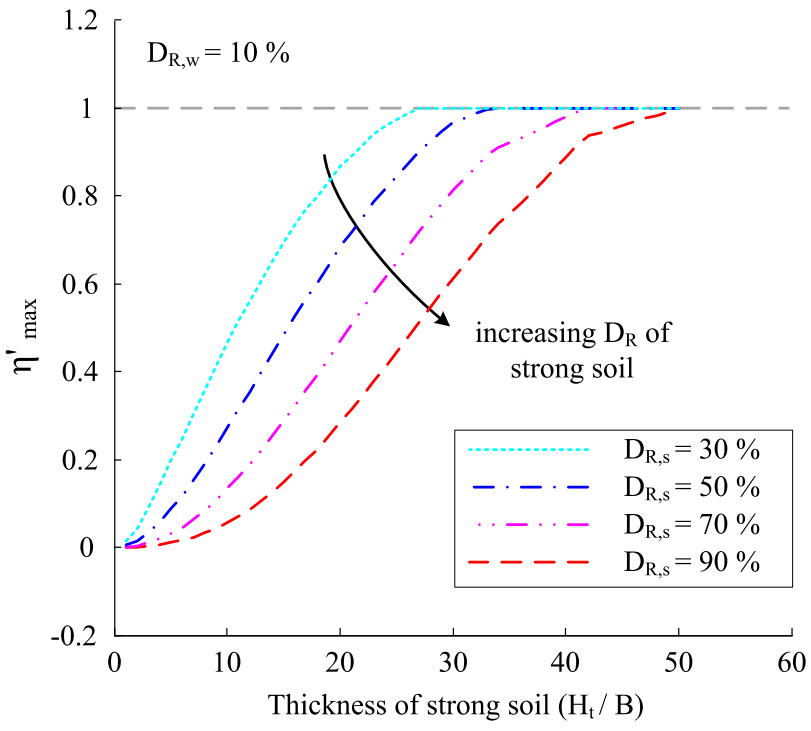

(a)

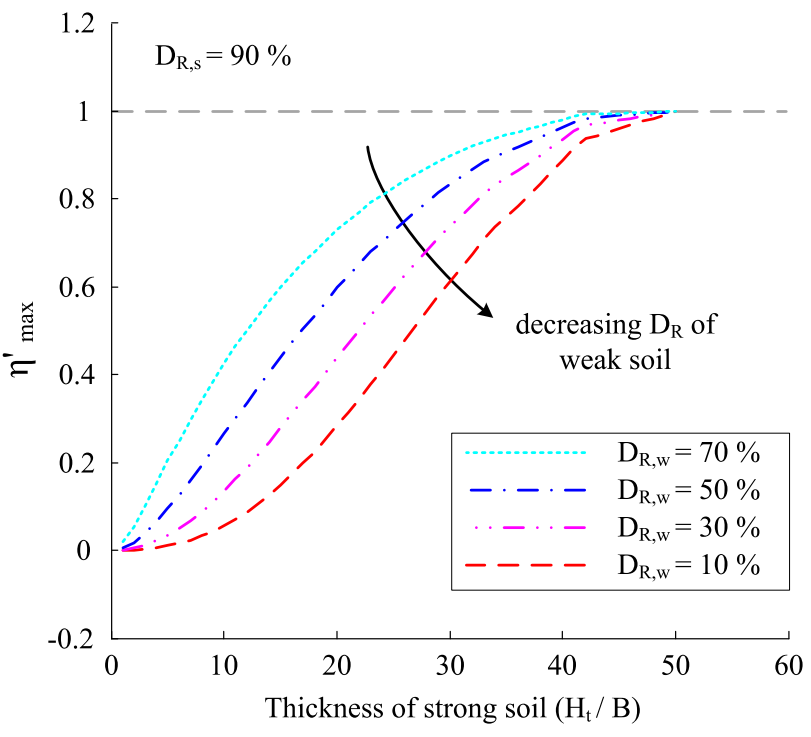

(b)

FIG. 14. Strong soil within weak soil: variation of the maximum resistance ratio $\eta_{\max }^{\prime}$ with the thickness of the thin-layer: (a) varying $D_{R}$ in strong soil; (b) varying $D_{R}$ in weak soil 
FIG. 15. Resistance ratio curves for thin-layer of weak soil $\left(D_{R}=10 \%\right)$ sandwiched by soils with $D_{R}=90 \%$ ), with variation of $H_{t} / B$ from 5 to 25 


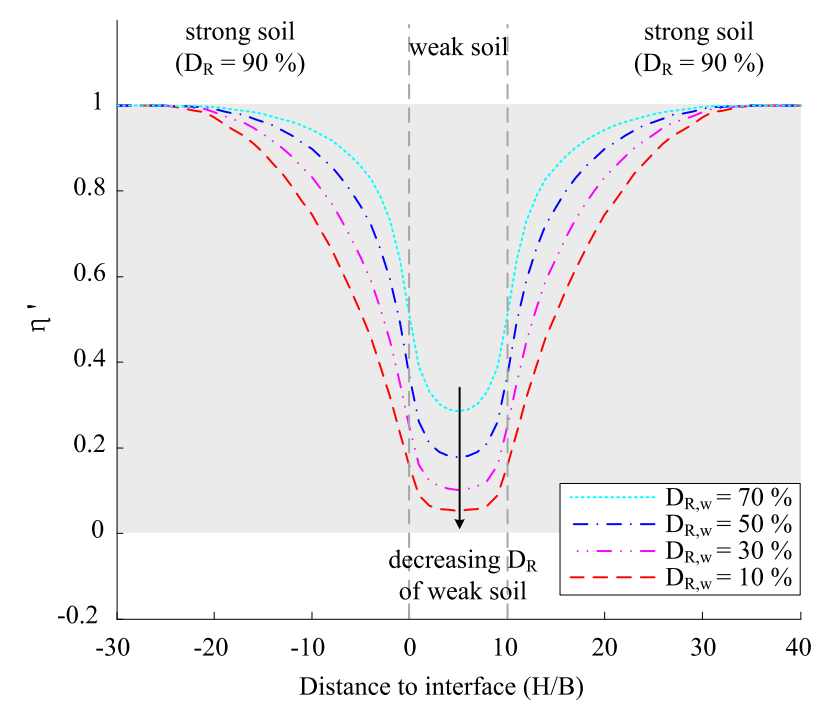

(a)

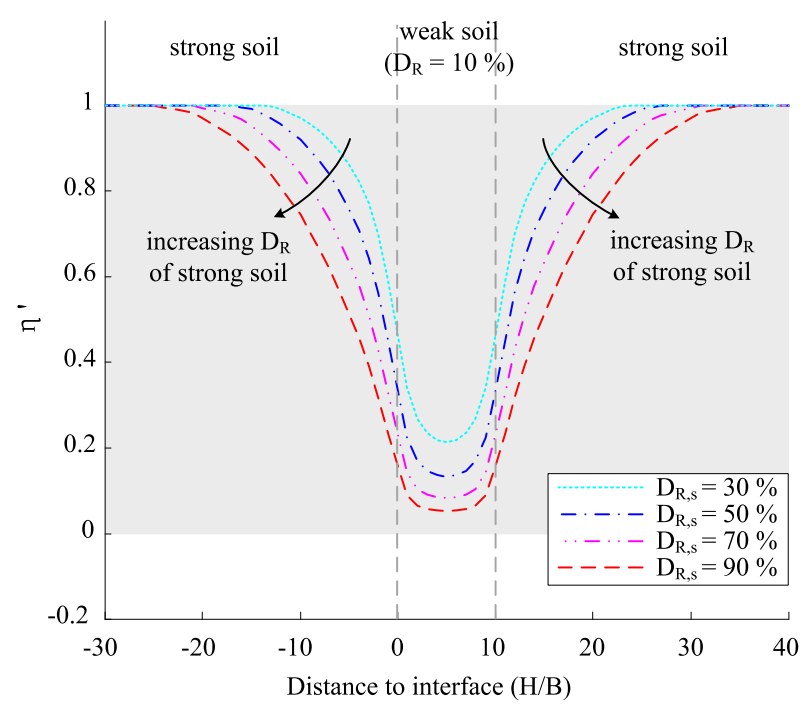

(b)

FIG. 16. Resistance ratio curves for thin-layer of weak soil $\left(H_{t} / B=10\right)$ : (a) varying $D_{R}$ in weak soil; (b) varying $D_{R}$ in strong soil 


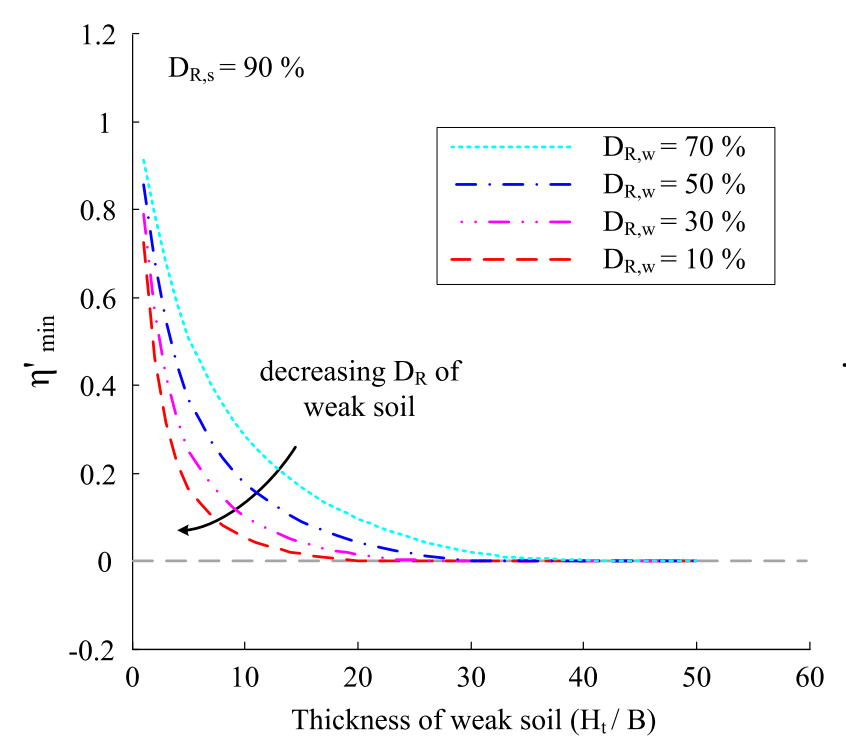

(a)

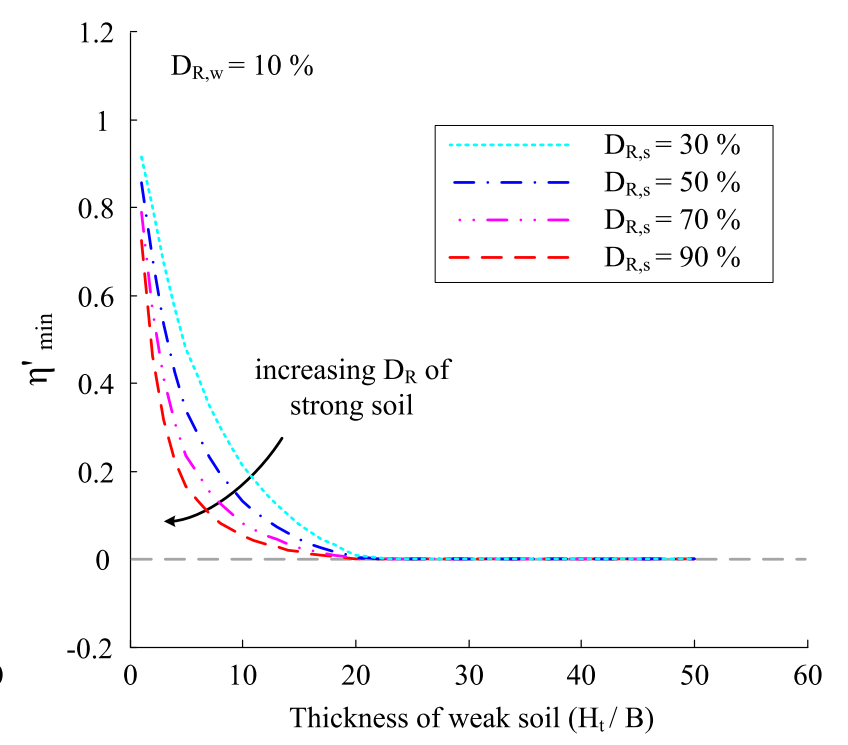

(b)

FIG. 17. Variation of the minimum resistance ratio $\eta_{\min }^{\prime}$ with the thickness of the thin-layer: (a) varying $D_{R}$ in weak soil; (b) varying $D_{R}$ in strong soil 


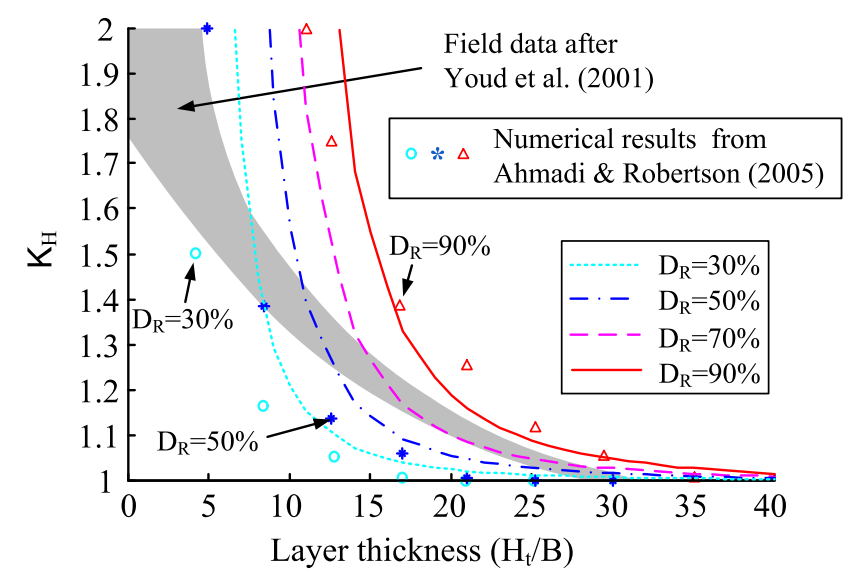

(a)

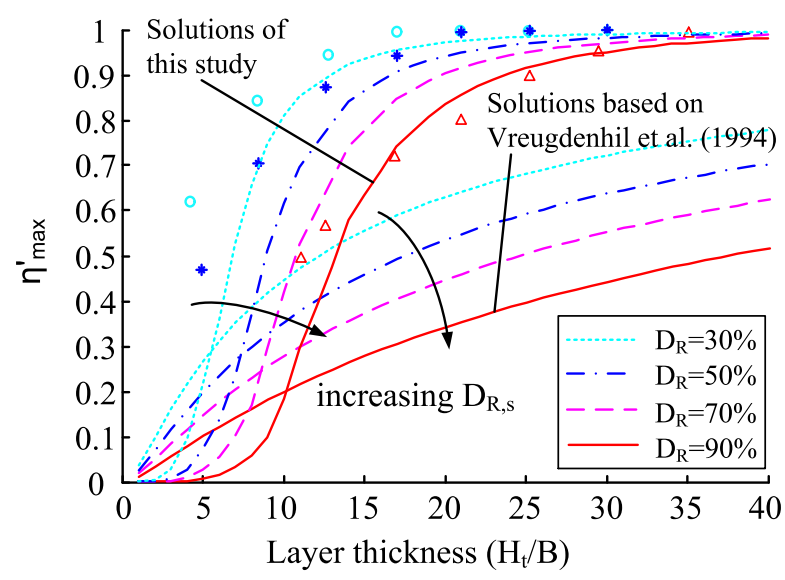

(b)

FIG. 18. Comparisons of the parameters for investigation of thin-layer effects: (a) $K_{H}$; (b) $\eta_{\max }^{\prime}$ 